\title{
La maladie de Pelizaeus-Merzbacher et une forme de paraplégie spastique liée à I'X sont toutes deux associées au gène PLP
}

Le gène responsable d'une forme compliquée de paraplégie spastique liée au chromosome $\mathrm{X}$ vient d'être identifié [1]. Il s'agit du même gène que celui déjà tenu pour responsable d'une autre affection neurologique, cliniquement très différente : la maladie de Pelizaeus-Merzbacher.

La maladie de Pelizaeus-Merzbacher (PMD, décrite en 1910) débute dès les premiers mois de la vie. C'est une maladie neurologique qui évolue progressivement vers une invalidité totale et un retard mental sévère [2]. A ce jour, treize mutations, toutes différentes, du gène PIP (Protéine Protéolipide), situé en $\mathrm{Xq} 22$, ont été décrites dans plusieurs formes de maladie de Pelizaeus-Merzbacher ([3-6], $\mathrm{m} / \mathrm{s}$ $n^{\circ} 1$, vol. 6, p. 70).

Les paraplégies spastiques représentent une forme de paralysie des membres inférieurs par atteinte du système nerveux central. Elles sont caractérisées par une très grande hétérogénéité clinique, certaines formes débutant dès l'enfance, d'autres pendant l'adolescence, et d'autres enfin à l'âge adulte. La sévérité de l'affection est très variable d'un patient à l'autre. Parmi les différentes formes de paraplégies spastiques, certaines ont une transmission liée au chromosome $\mathrm{X}$. On en distingue une forme pure et une forme compliquée dans laquelle, à la paraplégie spastique, s'associent un nystagmus, une ataxie, une atrophie optique et parfois un retard mental [7]. Notre démarche a été purement génétique: nous cherchions à préciser la localisation du gène responsable d'une forme compliquée de paraplégie spastique liée au chromosome X (SP(i2), grâce à une grande famille française comprenant onze garçons atteints de cette affection. Or, nous avons observé que le plus petit intervalle génétique contenant le gène $S P($ 2 2 contenait aussi le gène codant pour la protéine protéolipide PLP. Nous avons alors fait l'hypothèse que le gène $P L P$, déjà responsable d'une affection neurologique, la maladie de Pelizaeus-Merzbacher, pouvait également être en cause dans cette forme compliquée de paraplégie spastique liée à l'X. Le séquençage de la totalité de la région codante du gène $P L P$ d'un garçon atteint de paraplégie spastique a permis d'identifier une substitution dans le $3^{\mathrm{e}}$ exon du gène (Hisl39Tyr).

Il apparaît ainsi que SPG' et PMD, qui diffèrent à la fois par leur présentation neurologique et par leur évolution clinique, sont en fait des affections alléliques au locus PLP.
Comment des mutations du gène PLP peuvent-elles être responsables, à la fois, de la maladie de PelizaeusMerzbacher et de SP(;2? PIP code pour deux composants de la myéline du système nerveux central, PLP et DM20. Les deux isoformes diffèrent par l'épissage alternatif de l'exon 3 dont les 105 dernières paires de bases sont retenues dans PIP et non dans DM20. La mutation Hisl39Tyr est située précisément dans cette deuxième partie de l'exon 3. La mutation His139Tyr produit une protéine PLP mutée alors que son isoforme DM20 est normale. Il est à noter qu'aucune mutation dans cette portion d'exon n'a été rapportée à ce jour dans la maladie de Pelizaeus-Merzbacher. DM20 semble jouer un rôle majeur dans la maturation des oligodendrocytes alors que PLP est cruciale dans la compaction de la myéline. La préservation d'une différenciation normale des oligodendrocytes dans SP(i2 et non dans la maladie de Pelizaeus-Merzbacher rendrait compte des différences phénotypiques entre ces deux affections alléliques. Ainsi, nous pensons que le phénotype $S P\left({ }^{2} 2\right.$ doit être l'équivalent du phénotype murin rumpshaker, caractérisé par une hypomyélinisation associée à des oligodendrocytes 
en nombre normal et à morphologie normale. Les autres mutants animaux considérés comme des modèles de la maladie de PelizaeusMerzbacher (souris jimpy, rat $m d$, chien shaking pup) présentent toujours des anomalies des oligodendrocytes associés à l'hypomyélinisation.

Sur le plan neurologique, ce travail permet de revoir la classification des affections dysmyélinisantes du système nerveux central. Sur le plan génétique, il s'agit d'un nouvel exemple d'affections alléliques à phénotype différent, comme le sont la maladie de Kennedy et l'insensibilité aux androgènes, le syndrome de Pearson et le syndrome de Kearnes-Sayre, la maladie de Charcot-Marie-Tooth type 1 et le syndrome de Déjerine-Sottas $(\mathrm{m} / \mathrm{s}$ $n^{\circ} 1$, vol. 10, p. 100-3)

P.S-V.

A.M.

1. Saugier-Veber P, Munnich A, Bonneau D, Rozet JM, I e Merrer M, Boespflug-Tanguv (). $\mathrm{X}$-linked spastic paraplegia and PelizaeusMerzbacher disease are allelic disorders at the proteolipid protein locus on chromosome Xq21-q22. Nature Genet 1994; 6: 257-62. 2. Boulloche J, Aicardi J. PelizaeusMerzbacher disease: clinical and neurological study. J Child Neurol 1986; 1 : 233-9.

3. Pham-Dinh D, Nussbaum JL, Popot JL, Boespflug-Tanguy O, Landrieu P, Dautigny A. Mutations du gène codant pour les protéolipides de la myéline (PL.P et DM20) et dvsmyélinisations liées au chromosome $\mathrm{X}$. médecine/sciences $1992 ; 8: 664-72$.

4. Pratt VM, Kiefer JR, Lahdetie J, Schleutker J, Hodes ME, Dlouhy SR. Linkage of a new mutation in the proteolipid protein (PL.P) gene to Pelizaeus-Merzbacher disease (PMD) in a large fimnish kindred. Am J Hum Genet 1993; 52: 1053-6.

5. Iwaki A, Muramoto T, Iwaki T, Furumi H, Dario-del.eon ML, Tateishi J, Fukumaki Y. A missense mutation in the proteolipid protein gene responsible for Pelizaeus-Merzbacher disease in a Japanese family. $\mathrm{Hum} \mathrm{Mol}$ (jenet $1993 ; 2$ : 19-22.

6. Pham-Dinh D, Boesptlug-Tanguy O, Mimault C, Cavagna A, Giraud G, Leberre G; Lemarec B, Dautigny A. Pelizaeus-Merzbacher disease : a frameshift deletion/insertion event in the mvelin proteolipid gene. Hum Mol Genet 1993; 2 : 465-7.

7. Harding AE. Classification of the hereditary ataxias and paraplegias. Lancet 1983; 1:
Récepteurs de la vitamine D et ostéoporoses. L'ostéoporose pose un problème majeur de santé publique. Cette maladie dont la gravité croît avec l'âge, en particulier chez la femme ménopausée, est à l'origine de fractures, telles que celles de la hanche, aux conséquences dramatiques bien connues. Le risque de survenue de ces fractures est inversement corrélé à la densité osseuse, elle-même déterminée par des facteurs génétiques et non génétiques. Parmi ces derniers, les plus connus sont les déficits nutritionnels et hormonaux. Les facteurs génétiques, soupçonnés depuis plusieurs années, viennent d'être partiellement dévoilés dans l'étude d'une équipe australienne [1]. Cette équipe s'est intéressée au gène du récepteur de la vitamine $\mathrm{D}$ qui, compte tenu de son rôle dans l'absorption du calcium et le métabolisme osseux, représente un bon candidat potentiel pour être impliqué dans la maladie osseuse. La répartition de deux allèles $B$ et $b$ de ce gène $a$ été étudiée chez des jumeaux monozygotes et dizygotes; d'une part, l'allèle b est plus fréquemment retrouvé chez les individus à la densité osseuse la plus élevée; d'autre part, la densité osseuse est d'autant plus proche chez des jumeaux dizygotes qu'ils ont le même allèle du récepteur de la vitamine D. Cela constitue une bonne indication du rôle central joué par ces allèles dans la détermination de la densité osseuse. Plus surprenante est la constatation que cette corrélation dépend de la zone osseuse étudiée : très significative pour le rachis lombaire, elle l'est beaucoup moins pour le col du fémur. Dans une étude parallèle, les auteurs ont montré que la décroissance de la densité osseuse avec l'âge chez les femmes de génotype bb était nettement plus lente que celle observée chez les femmes BB. Ces travaux, une fois confirmés, pourraient aboutir à des applications thérapeutiques, en particulier dans la prévention de l'ostéoporose, mais les bases moléculaires restent incertaines. En effet, les allèles $B$ et b ne diffèrent que pour la partie 3' non codante de l'ARNm correspondant. L'insertion de cette séquence en aval du gène de la luciférase, dans des expériences de transfection cellulaire, conduit à des activités différentes du gène rapporteur. Curieusement, c'est l'allèle $B$ qui confère l'activité la plus importante, reflétant une plus grande stabilité de l'ARNm ou une transcription plus active du gène correspondant. On ne voit pas encore comment cette observation peut expliquer le rôle de l'allèle dans l'ostéoporose.

[1. Morrison NA, et al. Nature $1994 ; 367: 284-7$.

$\square$ Une compilation des gènes pathogènes comparés chez l'homme et chez la souris. Un article de revue vient d'être publié sur les homologues, chez la souris, des maladies héréditaires humaines. Il englobe 214 loci humains, cartographiés sur les chromosomes des deux espèces. Quarante-deux ont des variants pathologiques précisés actuellement dans les deux espèces. On peut, d'après les segments de séquence conservés, en prévoir au moins 50 autres. On y trouve aussi 66 homologues de protooncogènes et facteurs de croissance. Dix-huit loci pathologiques ou régions chromosomiques paraissent soumis à empreinte génomique.

[Searle AG, et al. J Med Genet 1994 ; 31: 1-19.] 